\title{
Cosmological bounce in Horndeski theory and beyond
}

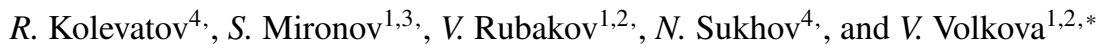 \\ ${ }^{1}$ Institute for Nuclear Research of the Russian Academy of Sciences, 60th October Anniversary \\ Prospect, 7a, 117312 Moscow, Russia \\ ${ }^{2}$ Department of Particle Physics and Cosmology, Physics Faculty, M.V. Lomonosov Moscow State \\ University, Vorobjevy Gory, 119991 Moscow, Russia \\ ${ }^{3}$ Institute for Theoretical and Experimental Physics, Bolshaya Cheriomyshkinskaya, 25, 117218 \\ Moscow, Russia \\ ${ }^{4}$ Department of Physics, Princeton University, Princeton, New Jersey 08544, USA
}

\begin{abstract}
We discuss the stability of the classical bouncing solutions in the general Horndeski theory and beyond Horndeski theory. We restate the no-go theorem, showing that in the general Horndeski theory there are no spatially flat non-singular cosmological solutions which are stable during entire evolution. We show the way to evade the no-go in beyond Horndeski theory and give two specific examples of bouncing solutions, whose asymptotic past and future or both are described by General Relativity (GR) with a conventional massless scalar field. Both solutions are free of any pathologies at all times.
\end{abstract}

\section{Introduction}

Cosmological bounce is one of the attractive ways to avoid the initial singularity implied by the conventional Big Bang theory. However, this type of cosmological solution requires the Null Energy Condition (NEC) to be violated and, hence, makes it necessary to consider a fairly exotic matter component. The general Horndeski theory suggests using a scalar field with the second derivatives in the Lagrangian as the source of NEC violation. Horndeski theories [1] are the most general scalar-tensor theories whose equations of motion are second order despite the presence of higher derivatives in the Lagrangian. An even broader class of theories was suggested in Ref. [2] and is usually referred to as "beyond" Horndeski ${ }^{1}$. General Horndeski and beyond Horndeski theories reveal similar features apart from the third order equations of motion in the latter case (still with no Ostrogradsky instabilities though).

The issue of stability has become the central one for a bounce in Horndeski theory. There are a lot of bouncing solutions suggested in the general Horndeski theory, which were free of obvious instabilities, but only during a certain period of time (to name just a few, see Refs. [47]). The situation with the construction of a complete and, at the same time, stable bounce remained a challenge. Finally, it has been shown in Ref. [8], that any spatially flat cosmological solution in the general Horndeski theory, including the bouncing one, is inevitably plagued by gradient instabilities, provided one considers the whole time of evolution of the Universe. The formulated no-go theorem suggests a loophole though, which requires either

*e-mail: volkova.viktoriya@physics.msu.ru

${ }^{1}$ Recently beyond Horndeski theories were generalized to the so called DHOST theories (see e.g. Ref. [3]). 
the fine-tuning or a strong coupling regime to be involved and both options are, generally, undesirable (see discussion in Sec. 3).

A healthy way to evade the no-go was proposed within beyond Horndeski theory: it was shown possible to have a spatially flat bouncing solution that is pathology-free throughout entire evolution. First examples of a completely healthy bounce were constructed within the EFT approach $[9,10]$ and provided inspiration for the following stable covariant constructions $[11,12]$.

Importantly, in Ref. [11] a peculiar issue of asymptotic behaviour of the bouncing model was addressed. Indeed, since (beyond) Horndeski theory is, generally, a theory of modified gravity, it is appealing to have a model with a bounce, which would reduce to GR with, say, a conventional massless scalar field in both asymptotic past and future. The main obstacle to having this kind of asymptotics is the so called $\gamma$-crossing. In a nutshell, $\gamma$-crossing denotes the moment when the denominator ${ }^{2}$, which is present in the coefficients of the quadratic action for scalar perturbations in the unitary gauge, vanishes. It was noted in Ref. [11] that $\gamma$ crossing is essential for constructing the bouncing solution with simple asymptotics. But, at a glance, $\gamma$-crossing causes the quadratic action for perturbations to have singular coefficients and, hence, has been avoided so far. Only recently Ref. [13] has explicitly shown that apparent divergences at $\gamma$-crossing are present only in the linearized equations, while the solutions are still regular. This fact is in full accordance with the analogous results obtained in the Newtonian gauge in Ref. [14]. Therefore, there is no reason to avoid $\gamma$-crossing.

However, it is important to note that $\gamma$-crossing does not help with evading the no-go theorem in the general Horndeski theory, modulo the loophole mentioned above. We discuss this issue in detail in Sec. 3. In this note by saying that the cosmological solution is "healthy" we imply that there are no pathologies (ghosts, gradient instabilities, etc.) arising throughout entire evolution, no fine-tuning involved and the potential strong coupling problem is avoided.

This note is based on the results presented in Refs. [11, 13] and is organised as follows. We gather the basic formulae for the linearized beyond Horndeski theory in Sec. 2 and pay special attention to the stability conditions. We give a detailed analysis of the no-go argument in Sec. 3. One of the main objectives of this note is to emphasise that it is possible to circumvent the no-go theorem only by going beyond Horndeski. Finally, in Sec. 4 we give the results for two complete, stable bouncing solutions with and without $\gamma$-crossing (these solutions were studied in Refs. [13] and [11]). We conclude in Sec. 5.

\section{Linearized theory and stability conditions}

We consider the scalar field theory with the following Lagrangian (mostly negative signature):

$$
\begin{aligned}
& S=\int \mathrm{d}^{4} x \sqrt{-g}\left(\mathcal{L}_{\mathcal{H}}+\mathcal{L}_{\mathcal{B H}}\right), \\
& \mathcal{L}_{\mathcal{H}}=F(\pi, X)+K(\pi, X) \square \pi-G_{4}(\pi, X) R+2 G_{4 X}(\pi, X)\left[(\square \pi)^{2}-\pi_{; \mu v} \pi^{; \mu \nu}\right]+ \\
& G_{5}(\pi, X) G^{\mu v} \pi_{; \mu \nu}+\frac{1}{3} G_{5 X}\left[(\square \pi)^{3}-3 \square \pi \pi_{; \mu \nu} \pi^{; \mu v}+2 \pi_{; \mu \nu} \pi^{; \mu \rho} \pi_{; \rho}^{\nu}\right], \\
& \mathcal{L}_{\mathcal{B H}}=F_{4}(\pi, X) \epsilon^{\mu v \rho}{ }_{\sigma} \epsilon^{\mu^{\prime} v^{\prime} \rho^{\prime} \sigma^{\prime}} \pi_{, \mu} \pi_{, \mu^{\prime}} \pi_{; \nu \gamma^{\prime}} \pi_{; \rho \rho^{\prime}}+ \\
& F_{5}(\pi, X) \epsilon^{\mu v \rho \sigma} \epsilon^{\mu^{\prime} v^{\prime} \rho^{\prime} \sigma^{\prime}} \pi_{, \mu} \pi_{, \mu^{\prime}} \pi_{; \nu v^{\prime}} \pi_{; \rho \rho^{\prime}} \pi_{; \sigma \sigma^{\prime}},
\end{aligned}
$$

where $\pi$ is the scalar field (sometimes dubbed generalized Galileon), $X=g^{\mu v} \pi_{, \mu} \pi_{, v}, \pi_{, \mu}=$ $\partial_{\mu} \pi, \pi_{; \mu v}=\nabla_{v} \nabla_{\mu} \pi, \square \pi=g^{\mu v} \nabla_{v} \nabla_{\mu} \pi, G_{4 X}=\partial G_{4} / \partial X$, etc. The general Horndeski theory corresponds to $\mathcal{L}_{\mathcal{H}}$.

\footnotetext{
${ }^{2}$ This denominator is denoted by $\Theta$ in the current work.
} 
To address the stability issue we consider perturbations about the spatially flat FLRW background. Generally, in (beyond) Horndeski theory there are two tensor degrees of freedom and only one dynamical degree of freedom in the scalar sector. In what follows we work in the unitary gauge, so the scalar field $\pi$ has vanishing perturbations $(\delta \pi=0)$. We parametrize the scalar and tensor perturbations of the linearized metric as follows:

$$
d s^{2}=(1+2 \alpha) d t^{2}-\partial_{i} \beta d t d x^{i}-a^{2}\left(1+2 \zeta \delta_{i j}+h_{i j}^{T}\right) d x^{i} d x^{j},
$$

where $h_{i j}^{T}$ denotes transverse, traceless tensor perturbations. Adopting the parametrization (2), the quadratic action for perturbations reads

$$
\begin{array}{r}
S=\int \mathrm{d} t \mathrm{~d}^{3} x a^{3}\left[\left(\frac{\hat{\mathcal{G}}_{\mathcal{T}}}{8}\left(\dot{h}_{i j}^{T}\right)^{2}-\frac{\mathcal{F}_{\mathcal{T}}}{8 a^{2}}\left(\partial_{k} h_{i j}^{T}\right)^{2}\right)+\left(-3 \hat{\mathcal{G}}_{\mathcal{T}} \dot{\zeta}^{2}+\mathcal{F}_{\mathcal{T}} \frac{(\nabla \zeta)^{2}}{a^{2}}-\right.\right. \\
\left.\left.-2 \mathcal{G}_{\mathcal{T}} \alpha \frac{\Delta \zeta}{a^{2}}+2 \hat{\mathcal{G}}_{\mathcal{T}} \dot{\zeta} \frac{\Delta \beta}{a^{2}}+6 \Theta \alpha \dot{\zeta}-2 \Theta \alpha \frac{\Delta \beta}{a^{2}}+\Sigma \alpha^{2}\right)\right]
\end{array}
$$

where

$$
\begin{aligned}
& \hat{\mathcal{G}}_{\mathcal{T}}=2 G_{4}-4 G_{4 X} X+G_{5 \pi} X-2 H G_{5 X} X \dot{\pi}+\dot{\pi}\left(2 F_{4} X \dot{\pi}^{2}+6 H F_{5} X^{2}\right)=\mathcal{G}_{\mathcal{T}}+\mathcal{D} \dot{\pi}, \\
& \mathcal{D}=2 F_{4} X \dot{\pi}+6 H F_{5} X^{2}, \\
& \mathcal{F}_{\mathcal{T}}=2 G_{4}-2 G_{5 X} X \ddot{\pi}-G_{5 \pi} X, \\
& \Theta=-K_{X} X \dot{\pi}+2 G_{4} H-8 H G_{4 X} X-8 H G_{4 X X} X^{2}+G_{4 \pi} \dot{\pi}+2 G_{4 \pi X} X \dot{\pi}-5 H^{2} G_{5 X} X \dot{\pi} \\
& -2 H^{2} G_{5 X X} X^{2} \dot{\pi}+3 H G_{5 \pi} X+2 H G_{5 \pi X} X^{2}+10 H F_{4} X^{2}+4 H F_{4 X} X^{3}+21 H^{2} F_{5} X^{2} \dot{\pi} \\
& +6 H^{2} F_{5 X} X^{3} \dot{\pi}, \\
& \Sigma=F_{X} X+2 F_{X X} X^{2}+12 H K_{X} X \dot{\pi}+6 H K_{X X} X^{2} \dot{\pi}-K_{\pi} X-K_{\pi X} X^{2}-6 H^{2} G_{4} \\
& +42 H^{2} G_{4 X} X+96 H^{2} G_{4 X X} X^{2}+24 H^{2} G_{4 X X X} X^{3}-6 H G_{4 \pi} \dot{\pi}-30 H G_{4 \pi X} X \dot{\pi} \\
& -12 H G_{4 \pi X X} X^{2} \dot{\pi}+30 H^{3} G_{5 X} X \dot{\pi}+26 H^{3} G_{5 X X} X^{2} \dot{\pi}+4 H^{3} G_{5 X X X} X^{3} \dot{\pi}-18 H^{2} G_{5 \pi} X \\
& -27 H^{2} G_{5 \pi X} X^{2}-6 H^{2} G_{5 \pi X X} X^{3}-90 H^{2} F_{4} X^{2}-78 H^{2} F_{4 X} X^{3}-12 H^{2} F_{4 X X} X^{4}- \\
& -168 H^{3} F_{5} X^{2} \dot{\pi}-102 H^{3} F_{5 X} X^{3} \dot{\pi}-12 H^{3} F_{5 X X} X^{4} \dot{\pi} .
\end{aligned}
$$

Lapse $(\alpha)$ and shift $(\beta)$ variables are non-dynamical, and variation of the action (3) with respect to them gives two constraint equations:

$$
\frac{\Delta \beta}{a^{2}}=\frac{1}{\Theta}\left(3 \Theta \dot{\zeta}-\mathcal{G}_{\mathcal{T}} \frac{\Delta \zeta}{a^{2}}+\Sigma \alpha\right), \quad \alpha=\frac{\hat{\mathcal{G}}_{\mathcal{T}} \dot{\zeta}}{\Theta} .
$$

So upon integrating out both $\alpha$ and $\beta$ in action (3), the quadratic action with two tensor and one scalar dynamical degrees of freedom takes the following form:

$$
S=\int \mathrm{d} t \mathrm{~d}^{3} x a^{3}\left[\frac{\hat{\mathcal{G}}_{\mathcal{T}}}{8}\left(\dot{h}_{i j}^{T}\right)^{2}-\frac{\mathcal{F}_{\mathcal{T}}}{8 a^{2}}\left(\partial_{k} h_{i j}^{T}\right)^{2}+\mathcal{G}_{\mathcal{S}} \dot{\zeta}^{2}-\mathcal{F}_{\mathcal{S}} \frac{(\nabla \zeta)^{2}}{a^{2}}\right],
$$

where the coefficients are

$$
\begin{aligned}
& \mathcal{G}_{\mathcal{S}}=\frac{\Sigma \hat{\mathcal{G}}_{\mathcal{T}}^{2}}{\Theta^{2}}+3 \hat{\mathcal{G}}_{\mathcal{T}}, \\
& \mathcal{F}_{\mathcal{S}}=\frac{1}{a} \frac{\mathrm{d} \xi}{\mathrm{d} t}-\mathcal{F}_{\mathcal{T}}, \\
& \xi=\frac{a \mathcal{G}_{\mathcal{T}} \hat{\mathcal{G}}_{\mathcal{T}}}{\Theta}=\frac{a\left(\hat{\mathcal{G}}_{\mathcal{T}}-\mathcal{D} \dot{\pi}^{\prime}\right) \hat{\mathcal{G}}_{\mathcal{T}}}{\Theta} .
\end{aligned}
$$


The squared speeds of sound for tensor modes $h_{i j}^{T}$ and curvature perturbation $\zeta$ are, respectively,

$$
c_{\mathcal{T}}^{2}=\frac{\mathcal{F}_{\mathcal{T}}}{\hat{\mathcal{G}}_{\mathcal{T}}}, \quad c_{\mathcal{S}}^{2}=\frac{\mathcal{F}_{\mathcal{S}}}{\mathcal{G}_{\mathcal{S}}} .
$$

Generally, to have a healthy solution (not necessarily a bouncing one), which is free of ghosts, gradient instabilities and superluminal modes, one has to impose the following constraints on the coefficients in the action (6):

$$
\hat{\mathcal{G}}_{\mathcal{T}}>\mathcal{F}_{\mathcal{T}}>0, \quad \mathcal{G}_{\mathcal{S}}>\mathcal{F}_{\mathcal{S}}>0
$$

We are now equipped to revisit the no-go theorem [8].

\section{No-go theorem in Horndeski theory and ways to evade it by going beyond Horndeski}

In this section we give a detailed explanation of the no-go argument formulated in Ref. [8] and describe the way to circumvent it by going beyond Horndeski. We also point out some new peculiar details about the behaviour of the metric perturbations at $\gamma$-crossing.

Basically, the no-go theorem states that in the general Horndeski theory the cosmological solutions, which are complete, healthy and stable during entire evolution do not exist. Here stability means that the solution is free of any kind of pathologies (ghosts, gradients instabilities, ets.) and it also implies that the system does not become strongly coupled at any time (i.e. none of $\hat{\mathcal{G}}_{\mathcal{T}}, \mathcal{F}_{\mathcal{T}}, \mathcal{G}_{\mathcal{S}}, \mathcal{F}_{\mathcal{S}}$ in eq. (6) tend to zero in either past or future or both).

Firstly, we would like to emphasise that the no-go argument in the general Horndeski theory does not rely on any specific choice of cosmological solution (bouncing scenario, Genesis, etc.). The theorem is, in fact, of a general character and only assumes the non-singular evolution of the scale factor $a$. Another important aspect is that the no-go considers stability during entire evolution of the Universe, from the infinite past to the infinite future. Therefore the theorem does not undermine numerous locally stable cosmological solutions constructed in Horndeski theory so far, but clearly shows that there are troubles with incorporating them in the general scheme of evolution, i.e. some pathologies are there anyway.

The main argument of the no-go theorem is based on the requirement of absence of gradient instabilities in the scalar sector, i.e. $\mathcal{F}_{\mathcal{S}}>0$, which may be cast in the following form, see definition (7b):

$$
\frac{\mathrm{d} \xi}{\mathrm{d} t}>a \mathcal{F}_{\mathcal{T}},
$$

As long as the scale factor $a$ is positive and bounded from below, which is true for any nonsingular cosmological scenario, and there are no gradient instabilities in the tensor sector $\left(\mathcal{F}_{\mathcal{T}}>0\right)$, the inequality (10) suggests that the function $\xi$ in eq. (7c) monotonously grows at all times.

What is more important, the constraint (10) implies that $\xi$ necessarily crosses zero at some finite moment of time. Indeed, it may be shown in a standard manner (see Refs. $[8,11]$ ) that the monotonously growing $\xi$ inevitably changes sign during the evolution. The latter fact implies inevitable zero-crossing in the case of continuous $\xi$. However, there is an option to consider a discontinuous $\xi$ (e.g. $\xi=-1 / t$ ), which changes sign by going through infinity and crosses zero only asymptotically, if at all. At least naively, it appears that $\xi$, behaving like this, safely meets the stability requirement (10), but in fact it is not. To avoid explicit crossing of zero, monotonously growing $\xi$ should asymptotically tend to either constant or zero at $t \rightarrow \pm \infty$, which results in $\dot{\xi} \rightarrow 0$ in both distant past and future. But to have vanishing 
$\dot{\xi}$ and satisfy the requirement (10), one has to allow $\mathcal{F}_{\mathcal{T}} \rightarrow 0$ at $t \rightarrow \pm \infty$, which is dangerous because of potential strong coupling at both time infinities. Thus, to satisfy the stability constraints $\hat{\mathcal{G}}_{\mathcal{T}}, \mathcal{F}_{\mathcal{T}}, \mathcal{G}_{\mathcal{S}}, \mathcal{F}_{\mathcal{S}}>0$ and, at the same time, avoid strongly coupled regime during entire evolution, $\xi$ necessarily crosses zero at some finite moment of time.

However, it appears that $\xi$ cannot cross zero in Horndeski theory in a healthy way. In the case of the general Horndeski theory $\hat{\mathcal{G}}_{\mathcal{T}}=\mathcal{G}_{\mathcal{T}}$ (see eq. (4a)), so according to the definition $(7 \mathrm{c})$

$$
\xi=\frac{a G_{\mathcal{T}}^{2}}{\Theta} .
$$

Thus, there are two options to make $\xi$ cross zero: 1) $\Theta \rightarrow \infty$, or 2) $\mathcal{G}_{\mathcal{T}} \rightarrow 0$. But infinite $\Theta$ means singularity in the Lagrangian, and $\mathcal{G}_{\mathcal{T}}$ tending to zero might result in a strongly coupled regime. This completes the no-go argument.

The situation is quite different in beyond Horndeski case though. Now $\hat{\mathcal{G}}_{\mathcal{T}}$ and $\mathcal{G}_{\mathcal{T}}$ in eq. (7c) have different values due to non-vanishing coefficient $\mathcal{D}$ (see eq. (4a)) and only $\hat{\mathcal{G}}_{\mathcal{T}}$ is constrained by the no-ghost requirement in action (6). So, in principle, it is possible to arrange the model with everywhere positive $\hat{\mathcal{G}}_{\mathcal{T}}$ and at the same time let $\mathcal{G}_{\mathcal{T}}$ safely cross zero. Hence, in beyond Horndeski theory the issue of $\xi$ crossing zero has a natural resolution thanks to the difference between $\hat{\mathcal{G}}_{\mathcal{T}}$ and $\mathcal{G}_{\mathcal{T}}$ and, hence, the no-go theorem is evaded. In the following section we demonstrate two specific examples of a bouncing solution in beyond Horndeski theory and explicitly show that they are free of any kind of pathologies throughout entire evolution.

Before we continue with the construction of a bounce in beyond Horndeski theory a comment is in order. In the no-go argument formulated above we have imposed no restrictions on the value of the coefficient $\Theta$ in eqs. (7) (except for the finiteness condition). At a glance, it appears that one has to avoid letting $\Theta$ cross zero, since it leads to the singular behaviour of both $\mathcal{G}_{\mathcal{S}}$ and $\mathcal{F}_{\mathcal{S}}$ in action (6). Moreover, both lapse and shift in eqs. (5) hit singularity when $\Theta=0$, which makes the stability analysis tricky in the unitary gauge. Therefore, in the general case $\gamma$-crossing appears dangerous.

However, it has been shown in Ref. [14] that there are no pathologies related to $\gamma$-crossing if one carries out the stability analysis in a Newtonian gauge. In particular, both the linearized equations and solutions for all scalar perturbations in the Newtonian gauge are non-singular for any value of $\Theta$ including zero. This finding implies, however, that in any other gauge there must be regular solutions for all scalar perturbations as well. And indeed, upon explicit verification, it was found in Ref. [13] that in the unitary gauge $\alpha, \beta$ and $\zeta$ do not develop any singularities at $\gamma$-crossing (see Ref. [13] for detailed derivations). The latter finding means that $\Theta$ can cross zero without causing any problems to the stability analysis in any gauge choice including unitary.

The motivation for having a healthy $\gamma$-crossing comes from attempts to construct a spatially flat bouncing solution within (beyond) Horndeski theory whose asymptotic past and future are described by General Relativity (GR) and a conventional massless scalar field. According to eq. (4d) $\Theta=H$ in the limit of General Relativity, where $K, G_{5}, F_{4}, F_{5} \rightarrow 0$ and $G_{4} \rightarrow \frac{1}{2}$. Since for a bouncing solution the Hubble parameter is negative at early times and positive at late times and, hence, crosses zero somewhere, regular $\gamma$-crossing is essential for restoring Einstein gravity with a conventional massless scalar field both before and after the bouncing stage (see Refs. [11, 14] for discussion). An explicit example of the bouncing solution of this type has been suggested recently in beyond Horndeski theory in Ref. [13] and is briefly described in the following section. 


\section{Healthy bounce with and without $\gamma$-crossing: examples}

In this section we consider two specific examples of bouncing solutions in beyond Horndeski theory with and without $\gamma$-crossing involved, which were suggested in Refs. [13] and [11], respectively. Both solutions are shown to be free of any kind of instabilities at all times. The model with everywhere positive $\Theta$ is arranged so that at late times the system asymptotes to GR with a conventional massless scalar field, while at early times there are no special requirements imposed on the theory. In the case of the bounce with $\gamma$-crossing the driving field $\pi$ reduces to a conventional massless scalar field and gravity tends to GR in both distant past and future.

Let us briefly outline the strategy of the solution construction employed in both cases (a full-blown reconstruction process of the Lagrangian is given in Refs. [11] and [13]). Without loss of generality we set the background scalar field $\pi$ to roll with constant speed:

$$
\pi(t)=t
$$

so that $X=1$. To construct the bouncing solution we choose the Hubble parameter and corresponding scale factor in the form:

$$
H=\frac{t}{3\left(\tau^{2}+t^{2}\right)}, \quad a(t)=\left(\tau^{2}+t^{2}\right)^{\frac{1}{6}}
$$

where the bounce occurs at $t=0$ and the parameter $\tau$ regulates the duration of the bouncing stage ( $\tau=1$ in Ref. [11]). The following procedure goes as follows: 1) we choose some of the functions in the Lagrangian (1), making sure that they reveal a required asymptotic behaviour and the stability conditions (9) are satisfied, 2) the leftover Lagrangian functions are determined from the background equations of motion with a corresponding choice of the Hubble parameter in (13). The only significant difference between the two solutions is whether $\Theta$ is allowed to cross zero or not (naturally, this fact has its influence on the initial choice of functions and their asymptotics). In both cases the aim is to have theories with no strong coupling involved at $t \rightarrow \pm \infty$.
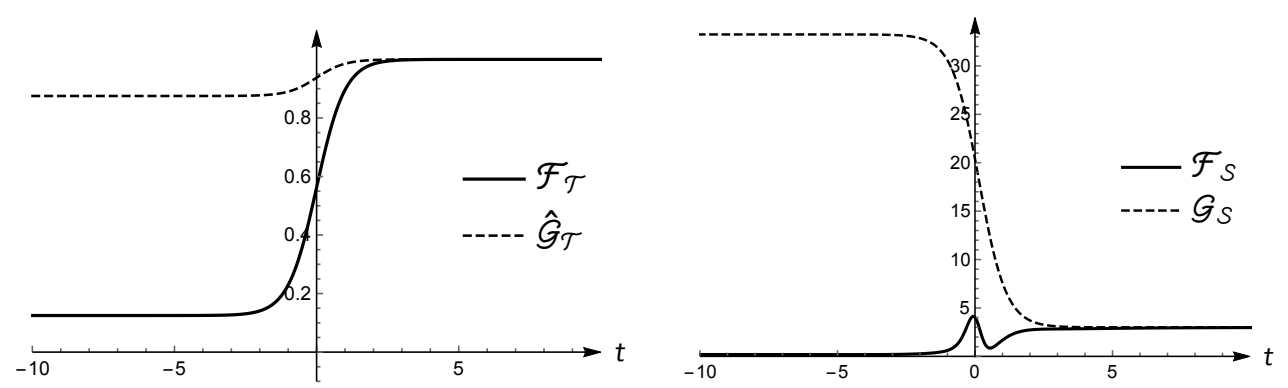

Figure 1. $\hat{\mathcal{G}}_{\mathcal{T}}, \mathcal{F}_{\mathcal{T}}, \mathcal{G}_{\mathcal{S}}, \mathcal{F}_{\mathcal{S}}$ for the bounce without $\gamma$-crossing. Note that $\mathcal{F}_{\mathcal{S}}$ is finite at $t \rightarrow-\infty$ : $\mathcal{F}_{\mathcal{S}} \rightarrow 0.193$.

Let us start with the results for the bounce without $\gamma$-crossing. The behaviour of the coefficients $\hat{\mathcal{G}}_{\mathcal{T}}, \mathcal{F}_{\mathcal{T}}, \mathcal{G}_{\mathcal{S}}, \mathcal{F}_{\mathcal{S}}$ is depicted in Fig. 1: all the variables are positive and bounded from below, which ensures that at both ends the theory is conventional (i.e. no strong coupling). The sound speed squared for both scalar and tensor sectors is given in the left panel of Fig. 2 and reveals the subluminal propagation of the corresponding perturbations. Thus, the constructed bouncing model is healthy and free of any kind of pathologies during entire evolution 
of the system. For completeness we show the behaviour of $\xi, G_{\mathcal{T}}$ and $\Theta$ in the left panel of Fig. 3: $\mathcal{G}_{\mathcal{T}}$ crosses zero and makes $\xi$ cross zero as well, while $\Theta$ is positive everywhere.
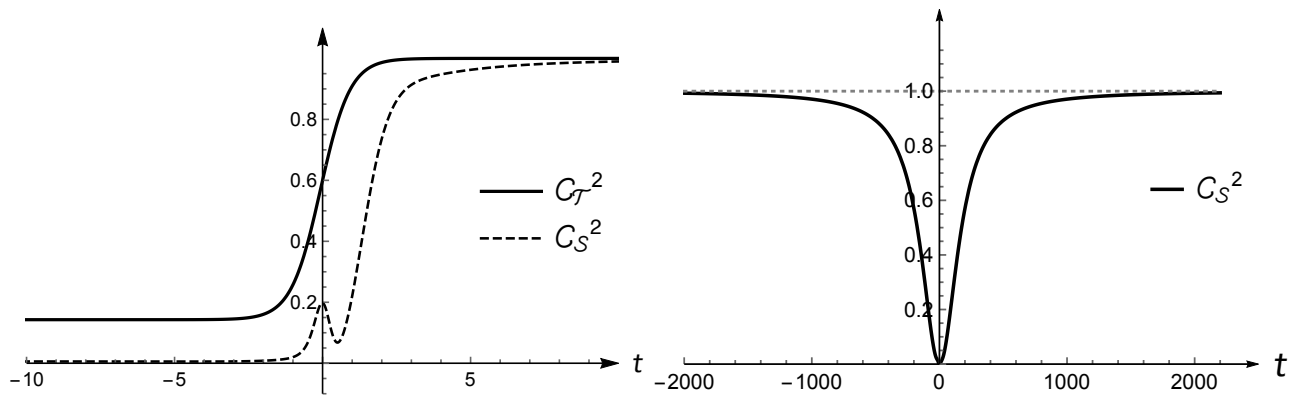

Figure 2. Sound speed squared for the scalar and tensor sectors. Left panel (bounce without $\gamma$ crossing): $c_{\mathcal{S}}^{2}$ is safely positive at $t \rightarrow-\infty: c_{\mathcal{S}}^{2} \rightarrow 0.006$. Both $c_{\mathcal{S}}^{2}$ and $c_{\mathcal{T}}^{2}$ tend to 1 at $t \rightarrow+\infty$. Right panel (bounce with $\gamma$-crossing): $c_{\mathcal{S}}^{2} \rightarrow 1$ as $t \rightarrow \pm \infty$ and is positive everywhere, $\min \left(c_{\mathcal{S}}^{2}\right) \approx 0.001$, while the tensor modes propagate at the speed of light $c_{\mathcal{T}}^{2}=1$.
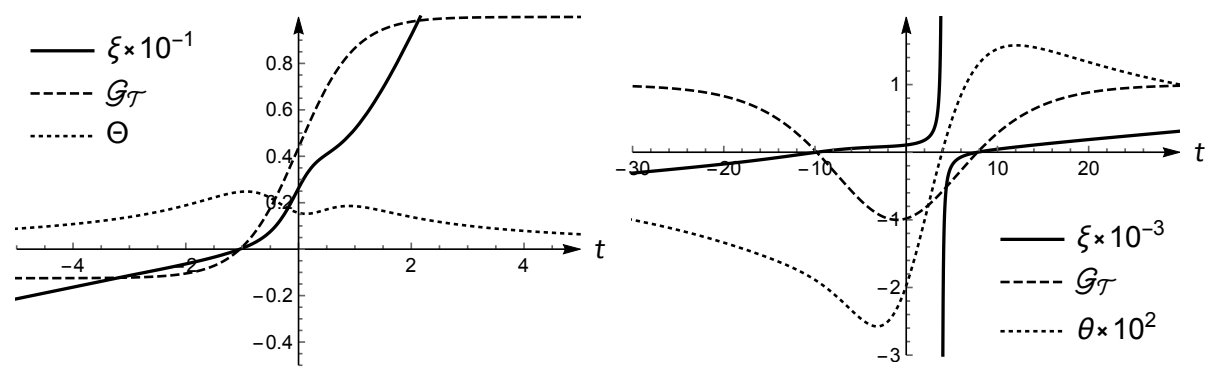

Figure 3. The behaviour of $\xi, \mathcal{G}_{\mathcal{T}}$ and $\Theta$. Left panel: $\xi$ crosses zero once at $t \approx-1.039$ thanks to $\mathcal{G}_{\mathcal{T}}$, while $\Theta$ is positive at all times, i.e. no $\gamma$-crossing. Right panel: $\xi$ crosses zero twice and is discontinuous when $\Theta$ is zero.

The coefficients $\mathcal{G}_{\mathcal{S}}$ and $\mathcal{F}_{\mathcal{S}}$ for the bounce with $\gamma$-crossing are shown in Fig. 4, while $\hat{\mathcal{G}}_{\mathcal{T}}=\mathcal{F}_{\mathcal{T}}=1$ due to a specific choice of Lagrangian functions in the set up. Both $\mathcal{G}_{\mathcal{S}}$ and $\mathcal{F}_{\mathcal{S}}$ are positive everywhere and infinite at the moment of $\gamma$-crossing. The peculiar feature of this bouncing model is that despite the singular behaviour of both $\mathcal{G}_{\mathcal{S}}$ and $\mathcal{F}_{\mathcal{S}}$ the linearized theory still can be studied in terms of the curvature perturbation $\zeta$, which is regular at all times (see Ref. [13]). The sound speed squared for the scalar sector is given in Fig. 2 (right panel) and is bounded from below. The tensor modes propagate at the speed of light $c_{\mathcal{T}}^{2}=1$. The right panel of Fig. 3 shows that in the case with $\gamma$-crossing $\xi$ is a discontinuous function and crosses zero twice but still due to behaviour of $\mathcal{G}_{\mathcal{T}}$.

Finally, let us give the asymptotic behaviour of both models at $t \rightarrow \pm \infty$. The Lagrangian of the bouncing model without $\gamma$-crossing has the following form at early times:

$$
\begin{aligned}
\left.\mathcal{L}\right|_{t=-\infty} & =C_{0} \cdot \frac{1}{\pi^{2}}+\left(\frac{1}{3}+C_{1}\right) \frac{(\partial \pi)^{2}}{\pi^{2}}+C_{2} \frac{(\partial \pi)^{4}}{\pi^{2}}+2 \frac{(\partial \pi)^{2}}{\pi} \square \pi-\frac{1}{16}(\partial \pi)^{2} R \\
& +\frac{1}{8}\left[(\square \pi)^{2}-\nabla^{\mu v} \pi \nabla_{\mu \nu} \pi\right]+\frac{1}{2} \epsilon^{\mu \nu \rho \sigma} \epsilon^{\mu^{\prime} v^{\prime} \rho^{\prime}}{ }_{\sigma} \nabla_{\mu} \pi \nabla_{\mu}^{\prime} \pi \nabla_{\nu v^{\prime}} \pi \nabla_{\rho \rho^{\prime}} \pi,
\end{aligned}
$$




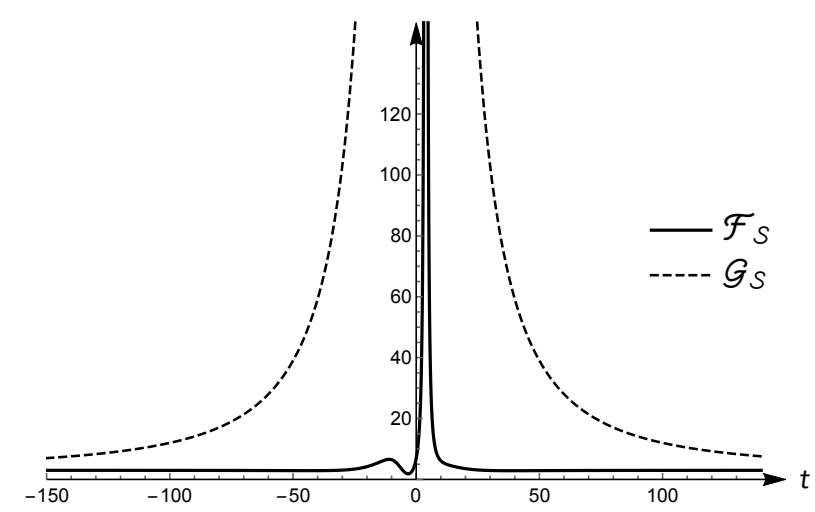

Figure 4. The coefficients $\mathcal{G}_{\mathcal{S}}$ and $\mathcal{F}_{\mathcal{S}}$ for the bounce with $\gamma$-crossing: both coefficients are bounded from below and diverge at $\gamma$-crossing.

where $C_{0}=2.43, C_{1}=-5.53$ and $C_{2}=1.06$ are model dependent constants. Note that eq. (14) does not reduce to GR in full accordance with the set up for this case. At late times, however, the Lagrangian of both bouncing models obtains a concise form

$$
\left.\mathcal{L}\right|_{t=+\infty}=-\frac{1}{2} R+\frac{1}{3} \frac{(\partial \pi)^{2}}{\pi^{2}}=-\frac{1}{2} R+\frac{1}{3}(\partial \phi)^{2},
$$

where $\phi=\ln (\pi)$. In the case of the bounce with $\gamma$-crossing, the Lagrangian has the form (15) at early times as well. So, in full accordance with our requirement, $\pi$ becomes a free massless scalar field and we are back to Einstein gravity in both asymptotic past and future provided that $\gamma$-crossing takes place at some point.

\section{Conclusion}

In this note we gave a detailed review of the no-go argument emphasising that the theorem can be evaded only by going beyond Horndeski theory. We described the results for two specific spatially flat bouncing solutions (with and without $\gamma$-crossing), which reduce to GR with a conventional massless scalar field in either one or both asymptotic states. Both models were shown to be complete and healthy during entire evolution.

\section{Acknowledgements}

Work by S. Mironov and V. Volkova was supported in part by the RFBR grant 18-32-00812 and the Foundation for the Advancement of Theoretical Physics and Mathematics "BASIS" grant.

\section{References}

[1] G. W. Horndeski, Int. J. Theor. Phys. 10, 363 (1974).

[2] J. Gleyzes, D. Langlois, F. Piazza and F. Vernizzi, Phys. Rev. Lett. 114 no.21, 211101 (2015)

[3] D. Langlois and K. Noui, JCAP 1602 no.02, 034 (2016) 
[4] D. A. Easson, I. Sawicki and A. Vikman, JCAP 1111021 (2011)

[5] Y. F. Cai, D. A. Easson and R. Brandenberger, JCAP 1208020 (2012)

[6] L. Battarra, M. Koehn, J. L. Lehners and B. A. Ovrut, JCAP 1407007 (2014)

[7] A. Ijjas and P. J. Steinhardt, Phys. Rev. Lett. 117 no.12, 121304 (2016)

[8] T. Kobayashi, Phys. Rev. D 94 no.4, 043511 (2016)

[9] Y. Cai, Y. Wan, H. G. Li, T. Qiu and Y. S. Piao, JHEP 1701090 (2017)

[10] P. Creminelli, D. Pirtskhalava, L. Santoni and E. Trincherini, JCAP 1611 no.11, 047, (2016)

[11] R. Kolevatov, S. Mironov, N. Sukhov and V. Volkova, JCAP 1708 no.08, 038 (2017)

[12] Y. Cai and Y. S. Piao, JHEP 1709027 (2017)

[13] S. Mironov, V. Rubakov and V. Volkova, arXiv:1807.08361 [hep-th]

[14] A. Ijjas, JCAP 1802 no.02, 007 (2018) 